\title{
A simple linear quadratic regulator (LQR) approach for active stabilization of mini rotors due to spinning dissipation
}

\author{
Abhro Mukherjee*, Satyabrata Das \\ Department of Electronics and Instrumentation Engineering, National Institute of Science and Technology, Berhampur- \\ 761008, Odisha, India
}

Corresponding Author Email: abhro456@gmail.com

https://doi.org/10.18280/ama_c.730406

Received: 23 April 2018

Accepted: 15 November 2018

\section{Keywords:}

internal damping, non-potential, shaft drawn power, critical speed, drive friction, stationary damping, linear quadratic regulator, piezo actuators and piezo sensors, PID controller, tool wandering

\begin{abstract}
Destabilizing effects due to rotating damper's in a gyrating or spinning systems is a very common phenomenon. Rotor's at speed higher than certain threshold values become unstable due to rotating damping forces generated by dissipation in rotor materials, coupling or due to friction in spline's and tool tip's. Presently the current methods are mostly passive and suitable for large or medium size rotor's but not quite applicable for small, mini or micro rotor systems. This paper uses an alternative technique to stabilize rotors of any size and description. The authors propose a piezo electrical type of actuating system for applying damping force to the rotating shaft by implementing a smart embedded coupling which rotates along with the rotor. The stabilization control was implemented by designing a simple state feedback Linear Quadratic Regulator whose gains were determined and applied to the rotor shaft through proposed smart embedded coupling.
\end{abstract}

\section{INTRODUCTION}

Rotors with internal damping are a very subtle dynamic to observe. The anti-symmetric nature of the circulating force causes the characteristics of this force to be non potential. Features of this non potential nature of the regenerative or the circulating force will be discussed in the preceding sections of this paper. This force causes the shaft to draw power from constant speed drive motor. Rotor systems which are subjected to these non-potential displacement dependent forces causes the rotor to destabilize. Some real life phenomena in rotor instability could be seen in elastic rotors with material or internal damping and also in forces resulting from hydrodynamic bearings and sometimes aerodynamic forces acting on elastic structures. Initially in the paper the nonpotential character of the circulating forces was established through extremely simple mathematical derivations taking into account the geometric transformation model between the fixed and rotating frame. One of the critical parameters to be determined ahead of designing the control law is the threshold or critical spinning speed of the rotor. In the later sections of the paper the critical or the threshold spinning speed was calculated by balancing both the regenerative and the dissipative power The threshold spinning speed of the rotor is extremely important for achieving rotor control as beyond this speed the rotor destabilizes. It has been observed that the rotor threshold spinning speed depends on its stiffness, external or the fixed damping and the internal damping of the system. The parameters over which the rotor threshold spinning speed depends are used later by the designer to establish control by modulating any one of these dependent parameters. Arguments have been given in favor to choose the rotor internal damping as the modulating parameter. It has been also shown that the rotor stiffness and the external or the fixed damping was discarded as the suitable parameter to be modulated by the proposed control law mainly for practical purposes and reasons which could be a hindrance in real time implementation of the controller and the actuator. Bond graph was used to model the complete rotor system at the initial stage. Bond graphs facilitate the creation of holistic models of dynamical systems belonging to multi energy domain. Since the complete sensing, control and actuation is all done in the rotating frame thus piezo or electromagnetic actuators are chosen as final actuating elements as because the size of the actuators can also be customized depending on the size of the rotor itself. Once the Bond-graph model of the system is formulated smart algorithms are used to convert a power driven bond graph model into a system state space description which is essential for designing the controller.

The discussions in this paper will focus on the real causes of instability due to material damping subjected to nonpotential nature of circulating force components in systems like elastic rotors, couplings and hydrodynamic bearing and how some earlier chosen techniques relied on some larger actuation system like the squeeze film dampers to arrest vibrations of relatively heavy rotors.

Authors further extended this work to reduce vibrations in face abrasive tools. Rotor systems with flat brittle materials like inorganic glasses or semiconductor substrata or bases used for preparing electronic wafer materials for polishing may cause tool wandering and unwanted vibrations due to presence of dry friction between the rotor tool tip and the polishing surface. It is therefore imperative to really think of strategies to reduce the tool tip wanderings and reduce vibrations as because the unwanted oscillations inflicted into the rotor system due to presence of dry friction could actually damage the materials being processed. Heavy mechanical dampers to arrest vibrations in rotor system with added tool kit were proposed earlier but due to complex features of such mechanical dampers the idea was not quite appropriate to be 
used for rotors which are small in size. Moreover incorporation of such mechanical dampers only modulate the parameter of stationary damping which is mostly applicable to medium or large sized grinders. Large mechanical dampers are not easily applicable to small size rotors.

The redemption of vibration in a face abrasive tool becomes more and more significant in the preparation of surfaces of brittle materials such as inorganic glasses and silicon materials.

\section{BRIEF LITERATURE REVIEW}

A wonderful paper describing 'Effects of diphase lubricants on Dynamics of rigid rotor' ASME [6] gave us an insight of physical dynamics of rotors in general and the effects of nonconservative type forces due to surprising behavior of these typical diphase lubricants and its destabilization effects on the rotors dynamics. The paper talked a lot about the nature and effects of these non-potential forces on the rotor and the need for an stabilization technique. In these above mentioned papers no control issues as such was discussed and moreover the results shown in the paper was particularly for large size rotors.

A paper, "An Investigation in to the Physics Behind the Stabilizing Effect of Two Phase Lubricants in Journal Bearings" Journal of vibration and control [18] discussed almost the same issues of as in [6] but more in details regarding the instabilities in the journal bearing due to anti-symmetric stiffness coefficients and also the general nature of bearing forces in the bearing system without much emphasize on the control strategies to stabilize such systems. But these papers were quite thorough giving strong evidence towards the nature of the bearing stiffness forces and its effects on the journal bearing as such. Paper [19] Kirillov O. N., "Gyroscopic Stabilization in the Presence of Non-conservative forces" Dokl. Math, 2007, also dealt with the techniques to mechanically stabilize systems affected by non conservative forces with heavy dampers once again not really suitable for rotors of small size and description. The instability issues of Tippe top dynamics because of internal damping of the system was given in the paper [21] "Dissipation-Induced Heteroclinic Orbits in Tippe Tops" 2008, SIAM Review. The dynamics of gyro pendulum shows exactly the same kind of unstable dynamics due to internal damping forces shown in paper [22] 'On Stability of Crandall Gyro-pendulum' Physics Letters. The above mentioned papers gave us a motivation to see the effects of these non-conservative type forces and its regenerative effects on a very small size pendulum type rotors and see if one can stabilize the rotors without using heavy dampers or squeeze film actuators with even more smart and elegant control strategies. Book chapter [1] MeirovitchL, "Analytical methods in vibration", N.Y Macmillan, and paper [2] was used to see if one can model these dynamical systems using power exchange dynamic graphs namely Bond-graphs which gave much better physical insight to dynamic systems than modeling it with plain differential equations. Paper [5] Rosenberg R.C. and Karnopp D., "Introduction to physical System Dynamics", Mc. Grew Hill, helped us to understand dynamics of physical system excited with non-potential fields it also gave us the mathematical insight of forces which are essentially non-potential in nature and how they affect the rotor dynamics and even addressed issues of the instability conditions in an hydrodynamic bearing system. One paper [9] "Theoretical and Experimental Studies on Squeeze Film Stabilizer for Flexible Rotor-Bearing Systems Using
Newtonian and Viscoelastic Lubricants" Jr. of Vibration and Acoustics, Transaction of ASME, used squeeze film dampers for large size rotor. Papers [7], [10] and [16] gave us an insight about the dynamics of piezo actuators and how it could be used as an actuating devise in a rotor model Book references [14] and [15] were used to understand the electrical and mechanical dynamics of the piezo-actuation system these references were also used to refer to the actuator parameters and its frequency responses. Book [17] gave us the complete insight of how easily bond graphs could be drawn of rotors with internal damping and one could also understand the algorithms to convert the bond graph model into a perfect state-space model whose matrices were later used to fine tune the controller. Some state feedback control strategies for stabilization of extremely large size rotors was studied [14], [15] but most of the control techniques were using conventional methods with complex control system. "Modeling and Simulation of Process Machine Interaction in Grinding systems" [23] this paper gave an overall insight that how dry friction forces between the rotor tool and the mating surface created a tool hunting and wandering effect within the rotor system however no real control strategy to overcome such instability was discussed. $\mathrm{PhD}$ thesis [24] was referred to understand the effects of rotor imbalances and external disturbances in the rotor system.

\section{ROTOR MODELING AND INSTABILITY ANALYSIS}

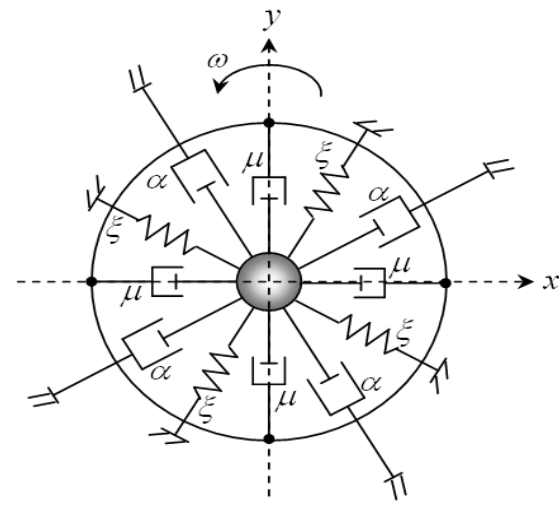

Figure 1. Distributed structure of the rotor damping and the rotor stiffness

One might choose the overall rotor parameters like the internal damping in the rotating frame, as $\mu=\frac{R_{i}}{2}$ such that its effective value in all directions is ' $\mathrm{R}_{\mathrm{i}}$ '. The external damping of the system is taken as $\alpha=\frac{R_{a}}{2}$ such that the effective damping coefficient in all direction is ' $\mathrm{R}_{\mathrm{a}}$ '. The stiffness coefficient $\varepsilon=\frac{K_{S}}{2}$, such that ' $\mathrm{K}_{\mathrm{s}}$ ' is experienced in all directions. If the overall dynamics of the rotor is considered, then the dynamic equation can be given as below

$m\left[\begin{array}{c}\ddot{X} \\ \ddot{Y}\end{array}\right]=-K\left[\begin{array}{l}\mathrm{X} \\ \mathrm{Y}\end{array}\right]-\left[\begin{array}{c}\dot{X} \\ \dot{Y}\end{array}\right] \mathrm{R}_{1}+\left[\begin{array}{cc}0 & \omega \mathrm{R}_{1} \\ -\omega \mathrm{R}_{1} & 0\end{array}\right]\left[\begin{array}{l}\mathrm{X} \\ \mathrm{Y}\end{array}\right]$

The above equation can also be written by the overall force components experienced by the rotor in ' $\mathrm{X}$ ' and ' $\mathrm{Y}$ ' directions of the shaft the governing equation in fixed reference frame can be seen from the equation below, 
$\left[\begin{array}{l}F x \\ F y\end{array}\right]=-K\left[\begin{array}{c}X_{f} \\ Y_{f}\end{array}\right]+R_{1}\left[\begin{array}{c}\dot{X}_{f} \\ \dot{Y}_{f}\end{array}\right]+\left[\begin{array}{cc}0 & -\omega R_{1} \\ \omega R_{1} & 0\end{array}\right]\left[\begin{array}{c}X_{f} \\ Y_{f}\end{array}\right]$

The dynamic force components can be seen as simple stiffness dependent forces, ordinary damping force proportional to $\dot{X}_{f}$ and $\dot{Y}_{\mathrm{f}}$ and finally the last component of the force which is of special significance. This component of the force can also be called as the regenerative force or the circulating force which exhibits a special feature that it is solely responsible for creating instability in the rotor system due to its anti symmetric nature

One might take this asymmetric force component given below for further analysis.

$\left[\begin{array}{l}F_{x c} \\ F_{y c}\end{array}\right]=\left[\begin{array}{cc}0 & -\omega R_{1} \\ \omega R_{1} & 0\end{array}\right]\left[\begin{array}{l}X \\ Y\end{array}\right]$

The special characteristics of this circulating force component is that it cannot be actually derived from any potential components. One may then say that like most of the other force components found in nature this force is apparently non-potential $\bar{F}_{c} \neq-\bar{V} \Phi$ where the function $\phi(\mathrm{x}, \mathrm{y})$ can be defined as some potential function.

One may get a rigorous insight of the dynamic equation by considering the overall geometric transformation by connecting the velocities in the rotating frame with those of fixed reference frame. Further the relationship of the rotating frame to those of the fixed reference frame is considered non co-rotating but a momentary snapshot of the dynamics may be taken when the fixed and the rotating frame is just co-oriented and the equations below are written based on these assumptions,

$V_{f}=V_{r}+\omega \times r$
$V_{f}=V_{r}+\omega \hat{k} \times(x \hat{\imath}+\mathrm{y} \hat{\jmath})$

The above equation in the matrix notations will be given as,

$\left[\begin{array}{c}\mathrm{V}_{\mathrm{x}, \mathrm{f}} \\ \mathrm{V}_{\mathrm{y}, \mathrm{f}}\end{array}\right]=\left[\begin{array}{c}\mathrm{V}_{\mathrm{x}, \mathrm{r}} \\ \mathrm{V}_{\mathrm{y}, \mathrm{r}}\end{array}\right]+\left[\begin{array}{cc}0 & \omega \\ -\omega & 0\end{array}\right]\left[\begin{array}{l}\mathrm{X}_{\mathrm{r}} \\ \mathrm{X}_{\mathrm{r}}\end{array}\right]$

In terms of displacement components

$\left[\begin{array}{c}\dot{\mathrm{X}}_{\mathrm{r}} \\ \dot{\mathrm{Y}}_{\mathrm{r}}\end{array}\right]=\left[\begin{array}{c}\dot{\mathrm{X}}_{\mathrm{f}} \\ \dot{\mathrm{Y}}_{\mathrm{f}}\end{array}\right]+\left[\begin{array}{cc}0 & -\omega \\ \omega & 0\end{array}\right]\left[\begin{array}{c}\mathrm{X}_{\mathrm{f}} \\ \mathrm{Y}_{\mathrm{f}}\end{array}\right]$

Multiplying the above equation with ' $\mathrm{R}_{\mathrm{i}}$ ' the damper force vector component would be given as

$\left[\begin{array}{c}F_{x} \\ F_{y}\end{array}\right]=R_{1}\left[\begin{array}{c}\dot{X}_{f} \\ \dot{Y}_{f}\end{array}\right]+\left[\begin{array}{cc}0 & -\omega R_{1} \\ \omega R_{1} & 0\end{array}\right]\left[\begin{array}{c}\mathrm{X}_{f} \\ Y_{f}\end{array}\right]$

Due to the nature of the circulating force component one might also get a non vanishing curl from the said dynamics,

$\overline{\mathrm{V}} \times \overline{\mathrm{F}}_{\mathrm{c}}=\left(\frac{\partial}{\partial \mathrm{x}_{\mathrm{i}}} \hat{\imath}+\frac{\partial}{\partial \mathrm{y}_{\mathrm{j}}} \hat{\mathrm{\jmath}}\right) \times\left(-\omega \mathrm{R}_{\mathrm{i}} \mathrm{y} \hat{\imath}+\omega \mathrm{R}_{\mathrm{i}} \mathrm{x} \hat{\jmath}\right)=2 \omega \mathrm{R}_{\mathrm{i}} \hat{\mathrm{k}}$

This has a very important and a significant property as because the work done by the circulating component of this force ' $F_{c}$ ' will be path dependent. We can also say that the net work will be done if the point of applications traces a closed orbit. Therefore the net work done in tracing a closed orbit will be given as,

$$
\mathrm{W}_{\mathrm{c}}=\oint \overline{\mathrm{F}}_{\mathrm{c}} \cdot \mathrm{dr}=\iint_{=2 \omega \mathrm{R}_{\mathrm{i}} \mathrm{A}} \overline{\mathrm{V}} \times \overline{\mathrm{F}}_{\mathrm{c}} \cdot \mathrm{da}=\iint\left(2 \omega \mathrm{R}_{\mathrm{i}} \hat{\mathrm{k}}\right) \cdot(\mathrm{da}) \hat{\mathrm{k}}
$$

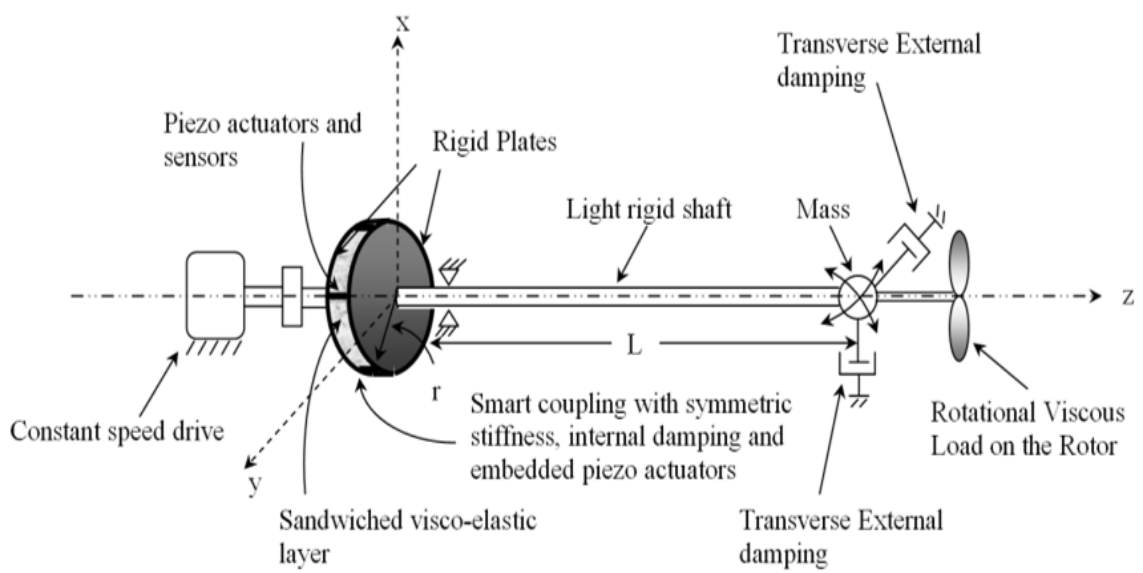

Figure 2. Smart embedded structure specially designed to actuate the rotor in rotating frame

If one considers the basic structure of the rotor from the figure 2 above, then the rotor rotates along the ' $z$ ' axis and it has flexibilities in both ' $\mathrm{X}$ ' and ' $\mathrm{Y}$ ' directions moreover the external damping is attached with the suspension system of the rotor. Both the isotropic stiffness and the internal damping has been taken as a lumped parameters coupled with the smart coupling for ease in modeling the system.

The integral part of the design includes a smart structure with attached sensor, controller and piezo type actuators within a single coupling. The essential feature of the smart coupling is that it rotates along with the rotor.

The complete sensing of position and velocity along with the control action which modulates the internal damping is done inside the smart structure.

\subsection{Tool Wandering analysis by adding tool kit element}




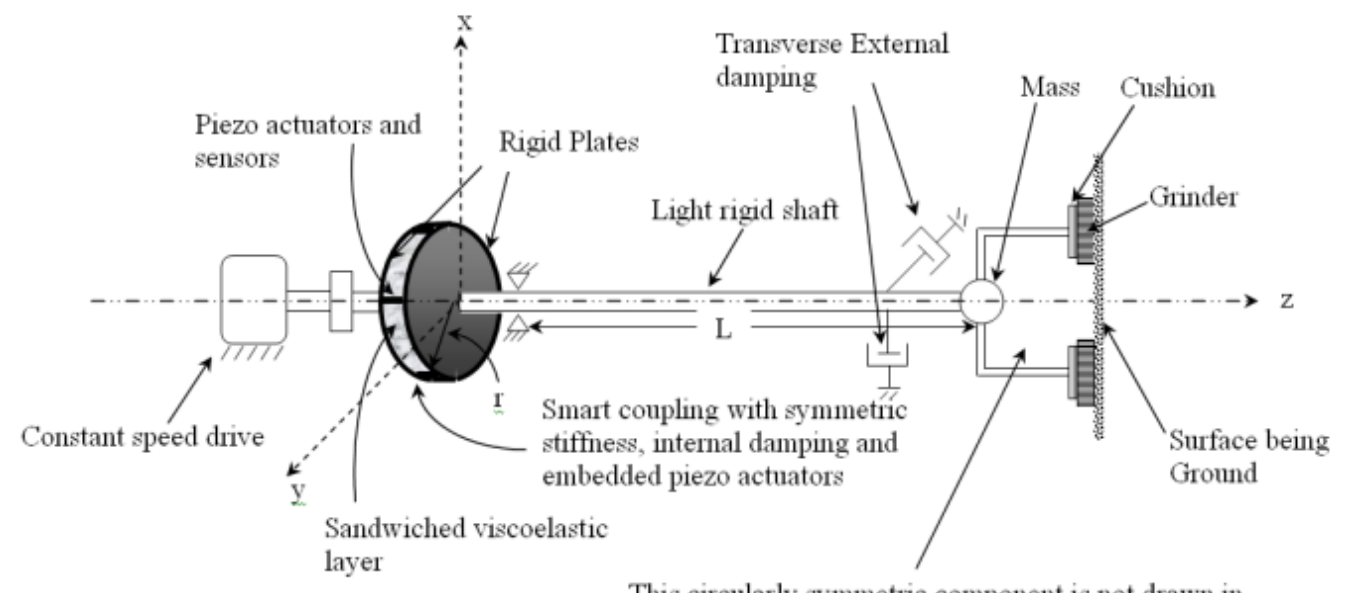

This circularly symmetric component is not drawn in perspective

Figure 3. Rotor system with embedded coupling and added tool kit element driven by constant speed drive motor

One may define the dry frictional force from standard tanh (Tan hyperbolic function) function given below.

$\tilde{F}=-F_{e r}\left\{\left(\tanh \left(z_{c} \dot{x}_{r}\right) \hat{\imath}\right)+\left(\tanh \left(z_{c} \dot{y}_{r}\right) \hat{\jmath}\right)\right\}$

The parameter ' $z_{c}$ ' can be considered yet another constitutive constant which depends on the materials of the mating surfaces. The nature of the dry frictional force can be represented in typically two regions of operation at low orbital speeds and also at higher orbital speeds.

One may overall contrive or try to see which of the following behavior listed below is truly shown by the dry frictional force element,

(a) Dissipative or anti dissipative

(b) Gyroscopic behavior

(c) Potential behavior

(d) Non potential or regenerative behavior

Mathematical operators describing the above behavioral pattern may be enlisted below

(a) $\quad d_{\bar{\nabla}} \times \bar{F}_{c}=\left(\frac{\partial}{\partial x_{i}} \hat{\imath}+\frac{\partial}{\partial y_{j}} \hat{\jmath}\right) \times$

$F_{c}$ (displacement curl)

$d_{\bar{\nabla}} \cdot \bar{F}=\left(\frac{\partial}{\partial x_{i}} \hat{\imath}+\frac{\partial}{\partial y_{j}} \hat{\jmath}\right) \times$

$F_{C}$ (displacement divergence)

(c)

(d)

$$
v_{\bar{\nabla}} \times F_{c}=\left(\frac{\partial}{\partial x_{i}} \hat{\imath}+\frac{\partial}{\partial y_{j}} \hat{\jmath}\right) \times F_{c} \text { (velocity curl) }
$$

$$
\begin{aligned}
& v_{\bar{\nabla}} \cdot \bar{F}=\left(\frac{\partial}{\partial x_{i}} \hat{\imath}+\frac{\partial}{\partial y_{j}} \hat{\jmath}\right) \times \\
& F_{c}(\text { velocity divergence })
\end{aligned}
$$

According to Stolz equation using the mathematical operators from (a) to (d) following conditions listed below will ensure the true behavior of dry frictional force.

(i)
There is a non-potential component if $d_{-} \bar{V} \times F$ is not zero.

Potential component of the force field occurs if $d_{-} \bar{V} . F$ is not zero.

Dissipative or anti dissipative force field occurs if $v_{-} \bar{V} \cdot \bar{F}_{c}$ is not zero.

Gyroscopic component of the force field occurs if $v_{-} \bar{V} \times \bar{F}$ is non-zero.

If $v_{-} \bar{V} \cdot \bar{F}$ is negative then it is dissipative in nature.
Using condition (iii) and given mathematical operator (d) by $v_{-} \bar{V} . F$ is negative which actually means

$$
v_{\nabla} \cdot F=-F_{e r} * z_{c} *\left\{\frac{1}{\cosh ^{2}\left(z_{c} * \dot{x}_{f}\right)}+\frac{1}{\cosh ^{2}\left(z_{c} * \dot{y}_{f}\right)}\right\}
$$

(the negativity of the term implies the nature of the force to be dissipative).

One may also find that the condition $v_{-} \bar{V} \times F=0$, implying a non gyroscopic nature.

The operator $d_{-} \nabla \times F$ gives the expression below

$$
d_{-} \bar{\nabla} \times F=-F_{e r} * z_{c} \omega\left\{\frac{1}{\cosh ^{2}\left(z_{c} * \dot{x}_{f}\right)}+\frac{1}{\cosh ^{2}\left(z_{\left.c^{*} \dot{y}_{f}\right)}\right.}\right\}
$$

It gives a non-zero value implying this force has a nonpotential component. Finally one can conclude that the dry friction force field to be dissipative, non-gyroscopic with no effective potential components. The analytical dry friction force has a peculiar property as it operates in two segments at lower speeds it operates within a linear region and at a higher orbital speed it remains in the saturation region.

One may further investigate with mathematical analysis how the dry frictional force allows the tool tip to oscillate or wander between the linear and saturated region causing instability in small size rotors which might be a hindrance for highly precise applications like polishing of thin film wafers of integrated circuits etc.

\subsection{Bond-graph model of the rotor system with and without the tool kit element.}

Due to the embedded coupling designed specially within the rotor. One may therefore model the entire dynamics in rotating frame.

The governing equation which then transforms a fixed reference frame to a rotating frame is given by

$$
\left[\begin{array}{l}
X_{P} \\
Y_{P}
\end{array}\right]=\left[\begin{array}{cc}
\cos \dot{\theta} & \sin \theta \\
-\sin \theta & \cos \theta
\end{array}\right]\left[\begin{array}{l}
X \\
Y
\end{array}\right]
$$

For doing the geometrical transformation one needs the dynamical model of the rotor which can be recalled as seen below 
$m\left[\begin{array}{c}\ddot{X} \\ \ddot{Y}\end{array}\right]=-K\left[\begin{array}{l}X \\ Y\end{array}\right]-\left[\begin{array}{l}\dot{X} \\ \dot{Y}\end{array}\right] R_{i}+\left[\begin{array}{cc}0 & \omega R_{i} \\ -\omega R_{i} & 0\end{array}\right]\left[\begin{array}{l}X \\ Y\end{array}\right]$

Here ' $\theta$ ' is the angle between the rotating and the fixed reference frame which has been used in the transformation

One might then finally get the transformed equation of the form below

$\left[\begin{array}{c}\dot{X}_{r} \\ \dot{Y}_{r}\end{array}\right]=\left[\begin{array}{cc}\cos \theta & \sin \theta \\ -\sin \theta & \cos \theta\end{array}\right]\left[\begin{array}{c}\dot{X}_{\mathrm{f}} \\ \dot{Y} \mathrm{f}\end{array}\right]+\left[\begin{array}{cc}-\sin \theta & \cos \theta \\ -\cos \theta & -\sin \theta\end{array}\right]\left[\begin{array}{c}X f \\ Y \mathrm{f}\end{array}\right] \dot{\theta}$

$$
\begin{aligned}
\dot{X}_{r}=(\cos \theta) \dot{X} \mathrm{f} & +(\sin \theta) \dot{Y} \mathrm{f} \\
& +(-\sin \theta X f+\cos \theta Y f) \dot{\theta}
\end{aligned}
$$

$$
\begin{aligned}
\dot{Y}_{r}=(-\sin \theta) \dot{X f} & +(\cos \theta) \dot{Y f} \\
& +((-\cos \theta) X f+(-\sin \theta) Y f)
\end{aligned}
$$

Since piezo actuators are available in small sizes therefore the choice of actuation system is done by embedding a set of piezo crystals properly aligned in the ' $\mathrm{X}$ ' and ' $\mathrm{Y}$ ' direction of the two degree of freedom rotor system. For a very small size rotor system one might embed the actuator within the rotating smart structure

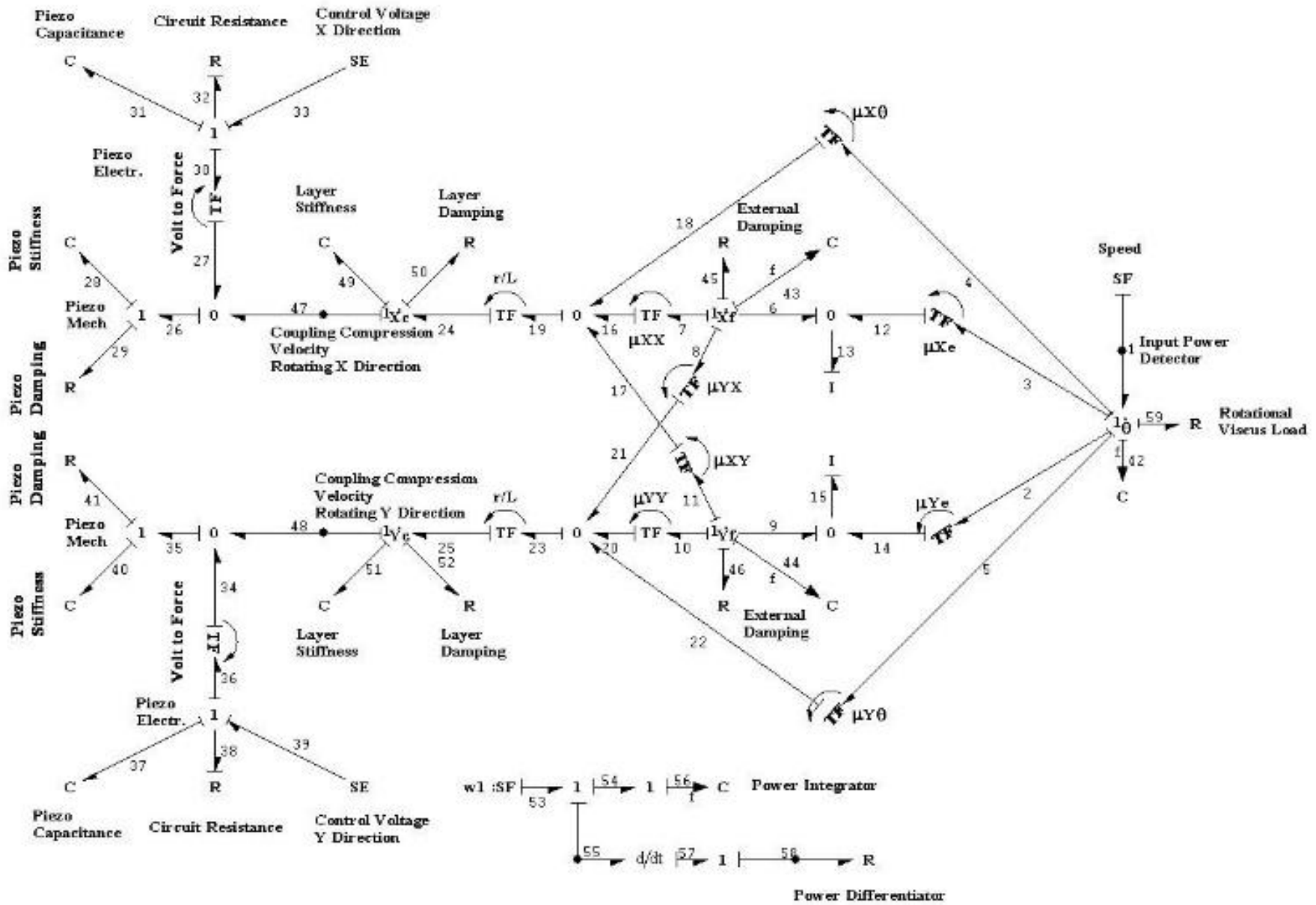

Figure 4. Bond graph model of the rotor system with smart embedded coupling

The equations (5) and (6) are mainly used to create the Bond graph model. Bond graphs are used to represent the entire dynamics of small scale rotor system as it is modeled completely by taking the power dynamics and which definitely gives a much better physical insight to the modeler.

The main transformation model from fixed to rotating frame is used as the base to create the power dynamics model using
Bondgraph.Once the dynamics has been properly modeled the piezo actuators are individually modeled and attached smartly on the rotating frame of the Bond graph model. Velocity sensors are fed in both ' $\mathrm{X}$ ' and ' $\mathrm{Y}$ ' directions of the rotating frame to sense and transfer data to the controller which may actually be considered also embedded within the embedded coupling structure rotating along with the rotor. 


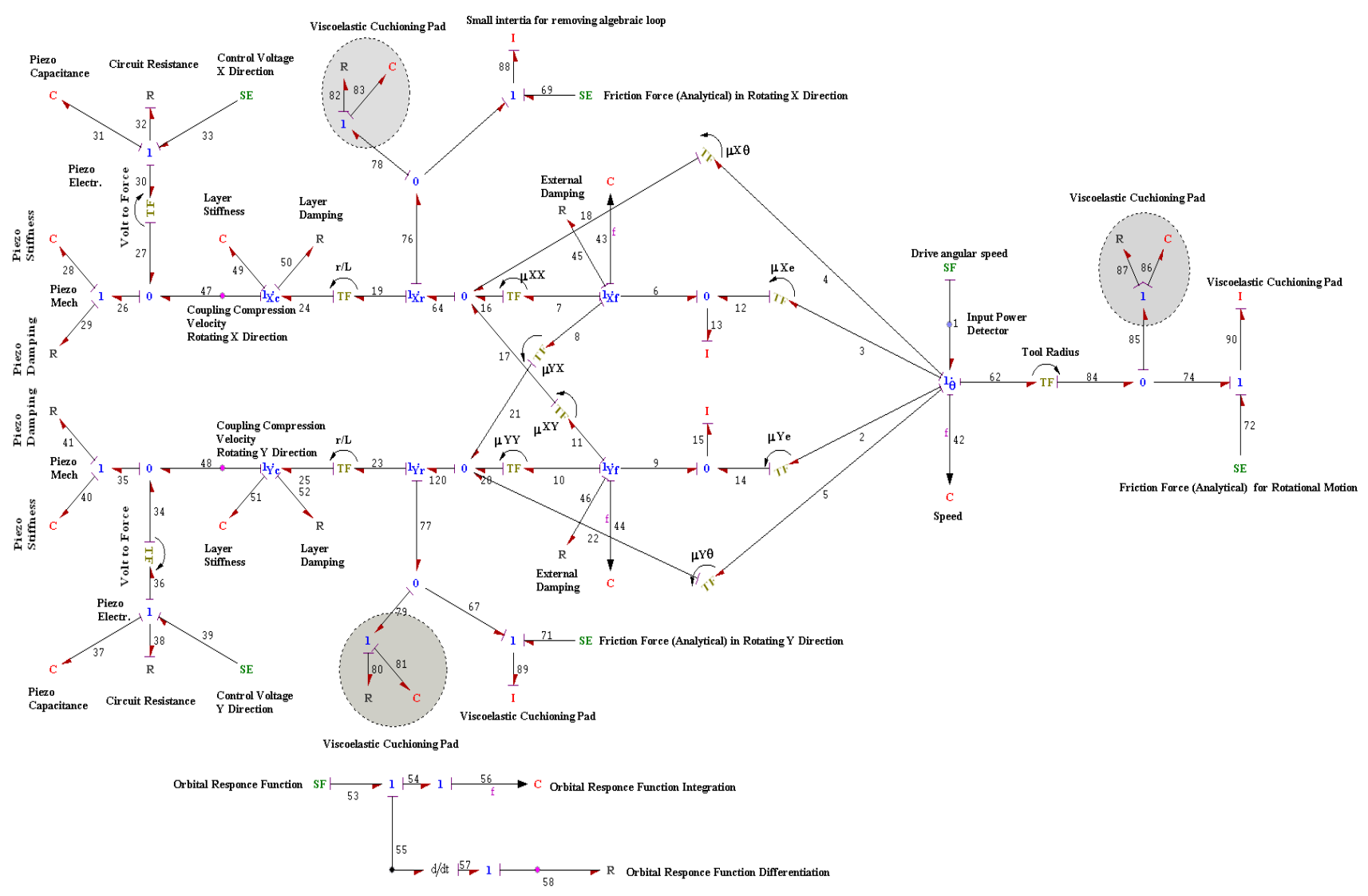

Figure 5. Bond graph model of the system with attached dry friction element between the rotor tool tip and the mating surface

Compared to the previous model which was without any additional tool kit element in this underlying model below fig 5 one may see that the dynamics was further extended by introducing elements of dry frictional force between rotor tool tip and the grinder or the mating surface. The behavior of the dry frictional force was initially mathematically defined and added to the model. One may introduce simple electromechanical piezo actuator in the model. Piezo actuating systems are preferred especially for small size rotor systems as using other actuation system needs more operating power which may outsize the rotor size itself.

Compared to the previous model which was without any additional tool kit in this model of fig (5) the dynamics was further extended by incorporating an additional attached face abrasive tool kit grinder.

\section{CONTROL OF SMALL SIZE ROTORS}

\subsection{Threshold speed calculation of rotor}

For the design of a suitable control it is obvious that the running speed ' $\omega$ ' of the rotor should be less than the threshold or the critical spinning speed 'wth'. At speeds greater than the threshold speed of the rotor the regenerative power becomes greater than the dissipative power and the rotor gets destabilized by consuming unwanted power from the constant speed drive motor thus there would be continuous growth of the power drawn by the shaft which actually renders it unstable .This is a highly unstable condition when the power drawn by the shaft increases monotonically. To design a suitable control strategy by making the running speed of the rotor not to cross the threshold speed one may calculate the relationship for the threshold critical spinning speed of the rotor. The smartest way to calculate the critical or threshold spinning speed of the rotor is to compute the regenerative power and the dissipative power and equate the two to address the verge of instability condition.

The relationship for the critical or the threshold spinning speed of the rotor is given by

$\omega_{t h}=\sqrt{\frac{k}{m}}\left(1+\frac{R_{a}}{R_{i}}\right)$

The rotor beyond this spinning speed tends to become unstable. Here the critical speed depends on the shaft natural frequency $\sqrt{\frac{k}{m}}$, external damping ' $\mathrm{R}_{\mathrm{a}}$ ' and the material or rotating damping ' $\mathrm{R}_{\mathrm{i}}$ '.

In this technique the regenerative energy per orbit is exactly balanced by the dissipative work done per orbit by the rotor.

At the critical condition or the balanced condition the regenerative work will be given by $W_{c}=2 \omega \mathrm{R}_{\mathrm{i}} \mathrm{A}$, moreover the system symmetry suggest that this orbit has to be identically circular in nature.

$\mathrm{W}_{\mathrm{c}}=2 \pi \omega \mathrm{R}_{\mathrm{i}} \mathrm{r}^{2}$

The dissipative work includes the damping factor $\left(\mathrm{R}_{\mathrm{a}}+\mathrm{R}_{\mathrm{i}}\right)$ and dissipative work done is given below. 
$\mathrm{W}_{\mathrm{d}}=(2 \pi \mathrm{r}) *\left(\omega_{\mathrm{n}} \mathrm{r}\right) *\left(\mathrm{R}_{\mathrm{i}}+\mathrm{R}_{\mathrm{a}}\right)$

At the verge of instability condition or the balanced condition of the rotor the regenerative power will be almost equal to the dissipative power.

Solving the condition $\mathrm{W}_{\mathrm{c}}=\mathrm{W}_{\mathrm{d}}$ one gets the relation already stated above

$\omega_{t h}=\omega_{n}\left(1+\frac{R_{a}}{R_{i}}\right)$

From the expression of the threshold speed it can be observed that the dependence of the critical speed is on natural frequency, the external damping and internal damping of the rotor. One might try to modulate any of these three parameters by the proposed control law to establish stability.

\subsection{Choice of the modulating parameter}

The threshold spinning speed of the rotor plays a definitive key role in designing a suitable control strategy. The prime objective of the controller is now to translate the threshold spinning speed ' $\omega_{t h}$ ' further away from the running speed of the rotor. In order to do that the controller has namely three parameters to choose from the condition of the threshold speed.

One may increase the shaft stiffness by feeding back shaft displacement. This way of modulation by the controller on the shaft stiffness is impractical and moreover there would be limitations up to which the stiffness could be increased in flexible rotors with limited number of actuators.

The design of the controller can be done in such a way such that one may increase the shaft stationary damping ' $R_{a}$ ' in order to shift the shaft critical spinning speed way beyond the running speed ' $\omega$ ' of the rotor. But some difficulties will be in this approach too, say if the range $\left(\omega-\omega_{t h}\right)$ (that is the difference of spinning and instability onset speed) is too high then higher feedback gains are to be given and since ' $\mathrm{Ra}$ ' already lies in the numerator of the critical speed relation one may find it difficult to generate such large scale external damping values by the controller. Fixing a high value of the external damping may pose certain difficulties as higher value of the external damping may increase the size of the actuation system itself.

If the size of the actuator itself becomes larger than the size of the rotor this would imply a serious draw back in the selection of the actuating element and fallback in the overall design. There might be severe constraints and problems to integrate such systems with the rotating shaft. There might also be additional problems arising to implement such systems due to high contact friction and wear in the actuator.

Therefore, for practical reasons one might modulate the internal damping ' $R_{i}$ ' One can re write the overall equivalent damping $R$ eqv $=R_{i}-R_{c}$ where $R_{\text {eqv }}$ is effective value of the rotating damping. Therefore the relationship for the threshold spinning speed is than given as $\omega_{\text {th }}=\omega_{n}\left(1+\frac{R_{a}}{R_{i}-R_{c}}\right)$ here 'Rc' is artificially created which depicts the actual control action. One may thus modulate the internal damping ' $\mathrm{R}_{\mathrm{i}}$ ' of the system by feeding back the forces proportional to the velocities in the rotating frame. The overall internal damping of the system may be reduced. This will translate ' $w$ th' way beyond ' $w$ ' and stabilization of the rotor may be achieved.

This stabilization strategy stands effective as it can be implemented in frame rotating with the shaft. Smart structures may be deployed along with some customized piezo-actuators. Piezo-actuators can be sealed down and miniaturized depending upon the rotor size.

One may sense the orbital area given as $X_{r}^{2}+Y_{r}^{2}$ but even better response function would be the velocity response function $\dot{X}_{r}^{2}+\dot{Y}_{r}^{2}$ as because the whirl orbiting speed is the natural frequency of the shaft without any damping.

Therefore, the overall control structure could now be given as,

$\mathrm{R}_{\mathrm{c}}^{\prime}=\left(\alpha * \mathrm{~V}_{\mathrm{amp}}^{2}+\beta * \int_{0}^{\mathrm{t}} \mathrm{V}_{\mathrm{amp}}^{2} \mathrm{dt}+\sigma * \frac{\mathrm{dV}_{\mathrm{amp}}^{2}}{\mathrm{dt}}\right)$

where $V_{a m p}^{2}=\left(\dot{X}_{r} * \frac{r}{L}\right)^{2}+\left(\dot{Y}_{r} * \frac{r}{L}\right)^{2}$

The following relation explains how the threshold running speed gets modulated

$\omega_{\text {th }}=\omega_{n}\left(1+\frac{R_{a}}{\left(R_{i}-\alpha P_{s}-\beta \int_{0}^{t} P_{s} d t-\gamma \frac{d P_{s}}{d t}\right)}\right)$

\subsection{Controller design using Riccati engine}

Initially before developing the control strategy the complete model was modeled using Bond graphs. Bond graphs are used extensively to model complex dynamic systems as it addresses the complexity in physical dynamics through power exchange and gives a much better physical insight to the modeler. Algorithms than can be furnished to convert bond graph model into exact state space model. The complexities in the rotor model lies in transforming the dynamics from fixed frame to rotating frame using transformation coefficients elegantly and then impending both the mechanical and electrical properties of the piezo actuation system within the model. Bond graph gives a very holistic insight and physical realization to the modeler. Just like one may convert transfer function model into signal flow graph similarly bond graphs can be converted into exact state space model by convertibility algorithms and calculations. In the current development of the controller we find out the system matrices and use the following design rules to implement the controller:

1. The entire state vector $\mathrm{x}(\mathrm{t})$ is available for feedback.

2. $\left[\begin{array}{ll}\mathrm{A} & \mathrm{B}\end{array}\right]$ is stabilized and $[\mathrm{A} \quad \mathrm{C}]$ is detectable.

3. $R=R^{T}>0$

Then

1. The linear quadratic controller is unique, optimal, full state feedback control law

$$
u(t)=-K x(t) \text { with } \mathrm{K}=R^{-1} B^{T} S
$$

That minimizes the cost, $J$, subjected to the dynamic constraint imposed by the open dynamics in the equation.

2. $\mathrm{S}$ is the unique, symmetric, positive semi definite solution to the algebraic Riccati equation

$S A+A^{T} S+C^{T} C-S B R^{-1} B^{T} S=0$

3. The closed loop dynamics arrived at by

$x(t)=(A-B k) x(t)$

It is guaranteed to be asymptotically stable.

4. The minimum value of the cost $\mathrm{J}$ is

$J=x_{0}^{T} S x_{0}$

In effect, the LQR (Linear quadratic Regulator) algorithm takes care of the tedious work done by the control systems engineer in optimizing the controller. However, the engineer still needs to specify the weighting factors and compare the 
results with the specified design goals. Often this means that controller synthesis will still be an iterative process where the engineer judges the produced "optimal" controllers through simulation and then adjusts the weighting factors to get a controller more in line with the specified design goals.

If the system is uncontrollable, then it is possible to drive $x(t)$ to zero in an arbitrary short period of time. This would require very large amount of control signal which from an engineering point of view are unacceptable. Large control signals will saturate actuator and if implemented in a feedback form will require high bandwidth designs that may excite un modeled dynamics. Hence, it is clear that there must be a balance between the desire to regulate the perturbations in the state to equilibrium and size of the control signal needed to do so. There are some assumptions in designing

1. The number of variables to keep small is equal to the number of controls. That is $\operatorname{dim}[z(t)]=\operatorname{dim}[u(t)]=m$.

2. The control weight is chosen such that $R=\rho \tilde{R}$ where $\rho$ is a positive scalar and $R=\tilde{R}^{T}>0$.

3. $\mathrm{G}_{\mathrm{z}}(\mathrm{s})$ is defined to be the square transfer function matrix between the variables we wish to keep small and the controls with the loop open, $z(s)=G_{z}(\mathrm{~s}) \mathrm{u}(s)$ where $G_{z}(\mathrm{~s})=C(s I-A)^{-1} B$, and $\mathrm{q}$ is the number of transmission zeros of $G_{z}(\mathrm{~s})$.

Adjusting $\rho$ directly influences the feedback control gain. When $\rightarrow \infty$, we speak of the LQR(Linear quadratic Regulator) controller as having low gain .

$u(t)=-\frac{1}{\rho} \tilde{R}^{-1} B^{T} S x(t) \rightarrow 0$

As $\rightarrow \infty$. Likewise, when $\rho \rightarrow 0$ we speak of the high gain controller since $u(t)$ will clearly become large

Calculations to determine the Controller gains

State Space Model:

$\ddot{o}+\frac{R i}{m} \dot{o}-\frac{(\omega R i-k)}{m} o=0$

$\left[\begin{array}{c}\dot{o}_{1} \\ \dot{o}_{2}\end{array}\right]=\left[\begin{array}{cc}0 & 1 \\ \frac{(\omega R i-k)}{m} & -\frac{R i}{m}\end{array}\right]\left[\begin{array}{l}o_{1} \\ o_{2}\end{array}\right]+\left[\begin{array}{l}0 \\ 1\end{array}\right] u$

Then the dynamic model before the state augmentation can be represented as,

$\dot{x}(t)=A x+B u(t)$,

$y=H x$, and $x_{0}\left(t_{0}\right)=x_{0}$,

The first augmented state to realize the internal controller may be represented as,

$\dot{X}_{r e f}(t)=N r-y=N r-H x$,

That is,

$\dot{X}_{\Sigma}=A_{\Sigma} X_{\Sigma}+B_{\Sigma} u+N_{\Sigma} r$

where $X_{\Sigma}=\left[\begin{array}{c}x(t) \\ x_{\text {ref }}(t)\end{array}\right] \in R^{c}$

Then the augmented state vector will be,

$A_{\Sigma}=\left[\begin{array}{cc}A & 0 \\ -H & 0\end{array}\right] \in R^{c \times c}$,

$B_{\Sigma}=\left[\begin{array}{c}B \\ 0\end{array}\right] \in R^{c \times m}, N_{\Sigma}=\left[\begin{array}{c}0 \\ N\end{array}\right] \in R^{c \times b}$

$\left[\begin{array}{l}\dot{e}_{1} \\ \dot{\mathrm{e}}_{2} \\ \dot{\mathrm{e}}_{3}\end{array}\right]=\left[\begin{array}{ccc}0 & 1 & 0 \\ \frac{(\omega R i-k)}{m} & -\frac{R i}{m} & 0 \\ 1 & 0 & 0\end{array}\right]\left[\begin{array}{l}e_{1} \\ \mathrm{e}_{2} \\ \mathrm{e}_{3}\end{array}\right]+\left[\begin{array}{l}0 \\ 1 \\ 0\end{array}\right]$

Kfeedback $=0.000017 \quad 0.1742 \quad 0.3162$

\section{DYNAMICS OF AN UNCONTROLLED ROTOR SYSTEM}

The rotor is rotated at a speed beyond ' $\mathrm{w}_{\mathrm{th}}$ '. There is no eccentricity within the rotor system. There is a initial inertia given to the rotor system in ' $\mathrm{X}$ ' direction. The plots below in Fig-6 shows that the orbit keeps increasing rapidly which means that the anti-symmetric non potential nature of the circulating force drives the shaft unstable and the plot below also shows that the shaft drawn power increases monotonically at a very fast rate.

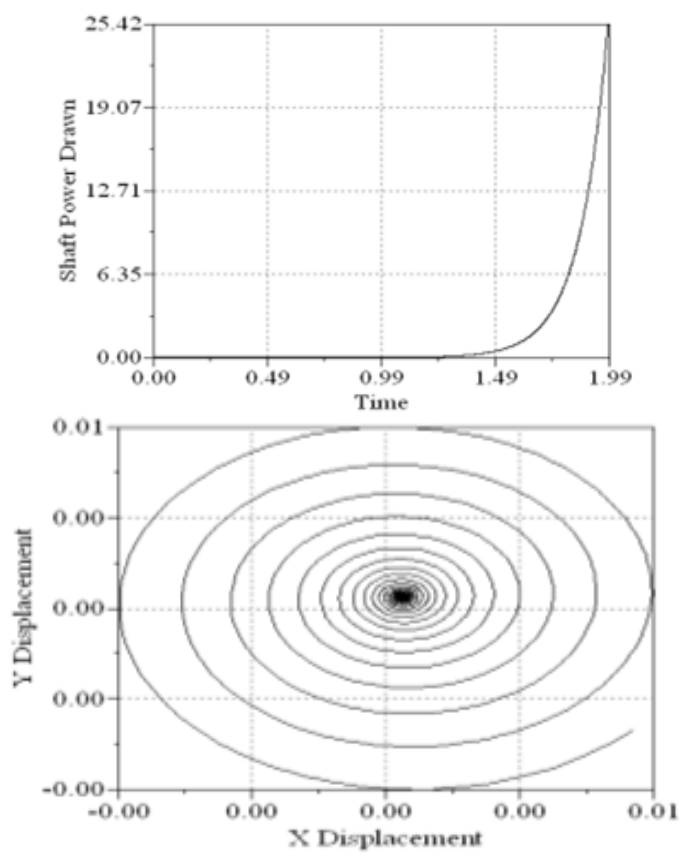

Figure 6. Rotor instability without the appended controller

\subsection{Stabilization of the rotor system with Linear Quadratic Regulator based controller without the added dry frictional element}

The parameters for this set of simulations are Mass $=0.1 \mathrm{~kg}$, Shaft stiffness $=1000.0 \mathrm{~N} / \mathrm{m}$, Stationary Damping $=0.2 \mathrm{Ns} / \mathrm{m}$, Rotating Damping=0.02 Ns/m, Driving Speed $=600$. The figures below show that the stabilizing action of the controller apparently stabilizes ' $\mathrm{Y}$ ' displacement and ' $\mathrm{X}$ ' displacement in both fixed and rotating frame. Fig(7a) is the threshold of instability speed which gets latched at a value.

Fig(7a) shows how the controller stabilized the shaft in ' $X$ ' direction and the convergence is in the order of $10^{-4} \mathrm{~mm}$. The rotor speed is $660 \mathrm{rad} / \mathrm{s}$ and the Riccati based stabilizing action lifts the threshold speed to $1127 \mathrm{rad} / \mathrm{s}$. This demonstrates the stabilization action. The figure $(7 \mathrm{~b})$ gives the complete phase trajectory of the rotor and from the simulation result it is clearly seen that the controller functions extremely well when the modulated part of the internal damping is less than the equivalent initial value of the internal damping but as soon as the modulated part of the internal damping becomes larger than the initial internal damping ' $\mathrm{Ri}$ ' the simulation result in 7(b) clearly shows that there is a start of reverse whirling of the rotor which the designed controller is unable to stabilize at the present moment therefore some modification of the controller is required by introducing an additional switching logic attached with the designed Linear Quadratic Regulating control. 

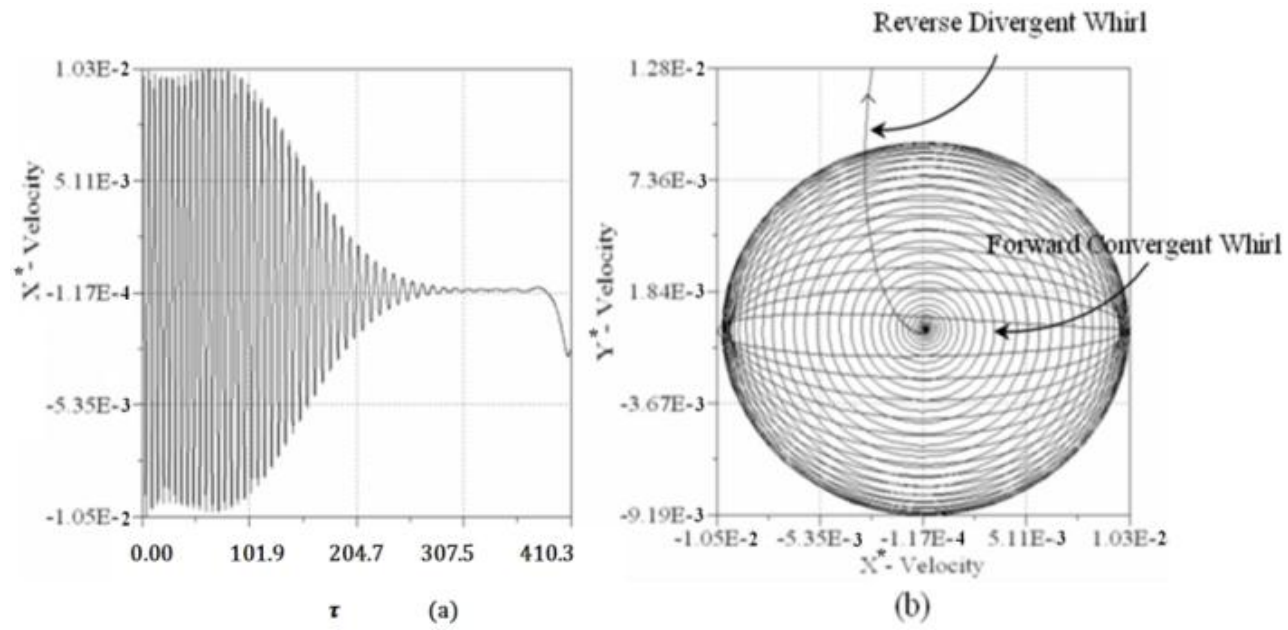

(b)

Figure 7. (a) and (b): Stabilized rotor with the appended LQR (linear quadratic regulator) control

\section{DYNAMICS OF AN UNCONTROLLED ROTOR SYSTEM}

The parameters chosen for this simulation are as given below.

SpeedRot $=600.0 \mathrm{rad} / \mathrm{s}, \mathrm{Ecc}=0.00001 \mathrm{~m}$, MassRot $=0.1 \mathrm{~kg}$, RadClp $=0.1 \mathrm{~m}$, Len $=0.1 \mathrm{~m}, \mathrm{Ra}=0.2 \mathrm{Ns} / \mathrm{m}$, Coupling Stiffness

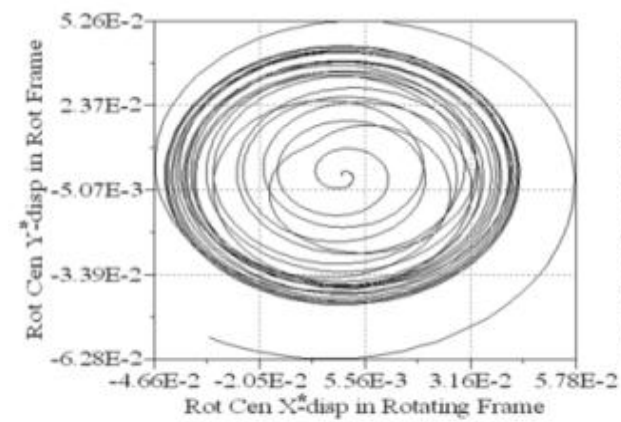

(a) referred to mass side displacement $=800.00 \mathrm{~N} / \mathrm{m}$, Coupling Damping referred to mass side velocity $=0.4 \mathrm{Ns} / \mathrm{m}$, Coef. Fric $=0.2$, Normal Force $=5 . \mathrm{N}, \mathrm{Zc}=1000.0$, Moment of Inertia $=0.01 \mathrm{kgm} 2$. Tool Radius $=0.001 \mathrm{~m}$, Tool Mass $=0.01 \mathrm{~kg}$, Tool

Inertia $=0.01 \mathrm{kgm} 2$. Tool Radius $=0.001 \mathrm{~m}$, Tool Mass $=0.01 \mathrm{~kg}$, Tool

Figure 8. (a) and (b): Instability and wandering effect shown by the rotor without any attached controller

In the above simulation that is in figure $8(\mathrm{a})$ and $8(\mathrm{~b})$ shows how the instability builds up due to the internal damping but additionally we can observe that how the instability builds up in a non-uniform Zig zag manner due to wandering effect of the dry frictional force in between the rotor tool tip and the mating surface.

\subsection{Simulation results with Linear Quadratic Regulator based active control and added dry friction element}

The parameters used for this simulation are as follow.
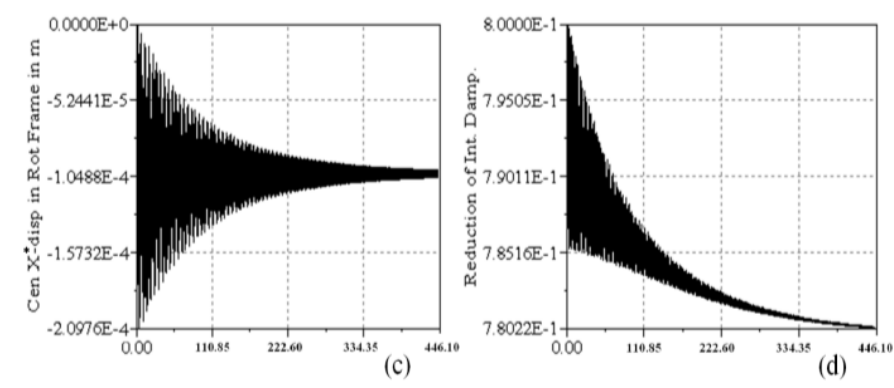

Figure 9. Stabilized rotor both in fixed and rotating frame using LQR (linear quadratic regulator) control and attached dry frictional element 
SpeedRot $=500.0 \mathrm{rad} / \mathrm{s}$, Ecc $=0.00001 \mathrm{~m}$, Mass of the Rot. $=0.1 \mathrm{~kg}, \operatorname{RadClp}=0.1 \mathrm{~m}$, Len $=0.1 \mathrm{~m}, \mathrm{Ra}=0.2 \mathrm{Ns} / \mathrm{m}$, Coupling Stiffness referred to mass side displacement $=800.00 \mathrm{~N} / \mathrm{m}$, Coupling Damping referred to mass side velocity $=0.4 \mathrm{Ns} / \mathrm{m}$, Coef. Fric $=0.2$, Normal Force $=5.0 \mathrm{~N}, \mathrm{Zc}=1000.0$, Tool Radius $=0.001 \mathrm{~m}$, Tool Mass $=0.01 \mathrm{~kg}$, Tool Moment of Inertia $=0.01 \mathrm{kgm}^{2}$. OrbResFunc $=2.0$, Cushion Pad Damping $=0.4 \mathrm{Ns} / \mathrm{m}, \mathrm{Kpad}=1000.0 \mathrm{~N} / \mathrm{m}$, Latched Initial Int. Damp Reduction, $\mathrm{Rc}=0.8$

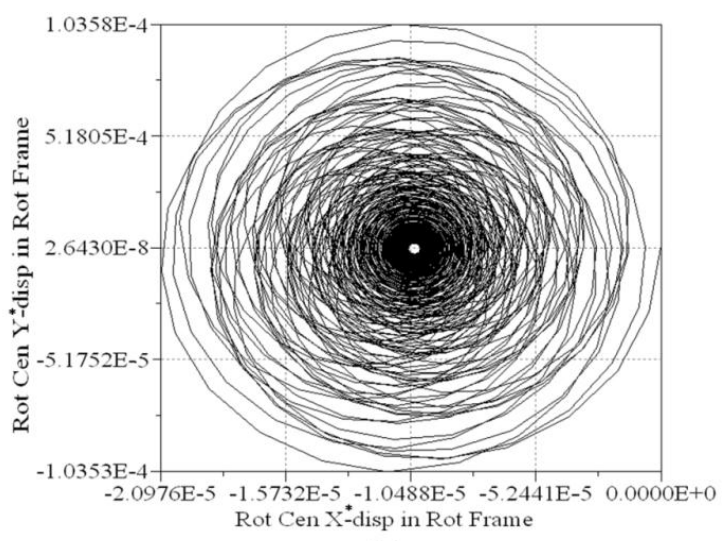

(a)

Figure 10. ' $\mathrm{X}$ ' and ' $\mathrm{Y}$ ' trajectory of stabilized rotor with added dry frictional element

Fig. (10) shows how the controller stabilized ' $\mathrm{X}$ ' and ' $\mathrm{Y}$ ' displacement smoothly with little or almost no wandering effect in both fixed and rotating frame as simulated by the symbols shakti software. Fig. (9) shows the stabilized phase trajectory of the rotor. This amplitude progressively reduces and then settles down to a small value even with the addition of dry frictional force acting in the rotor.

\subsection{Brief discussion on the simulation results}

The authors have designed a self-tuned controller for stabilizing extremely small size rotors with mass of about 0.1 $\mathrm{Kg}$ therefore not enough articles were found which addressed stabilizing issues within this size. Most of the other papers designed controllers for medium to much larger size rotors. Although the simulation results of present controller is comparable to other papers which used conventional controllers like Sliding mode and pole-placement but some improvement was seen over other optimal control techniques like Dynamic programming or Pontygrins method.The novelty of this current work was actually to use the orbital function $\dot{X}_{r}^{2}+\dot{Y}_{r}^{2}$ which reduced the original model with larger number of states and coupling into a more simplified model. This simplification made the controller tuning easier and much more realistic which will help the designer to implement it in a real time processor with reduced number physical states and sensors. Most of the current work made the controller design much murkier and less simplified by using larger number of internally coupled states. Therefore, decoupling the internally interacting states made the design quite complex.

\section{CONCLUSION}

This paper shows the destabilizing effect of spinning damping in a rotor system. There are different methods to stabilize such rotor systems but most of earlier methods are passive in nature using heavy squeeze film and larger mechanical dampers suitable for large or medium size rotors. This paper gives an active stabilization method to stabilize small to mini size rotors. The spinning damping and the actuation devices are all lumped to a smart coupling between the drive and the rotor.

When the rotor does not drive the load the shaft drawn power is a good measure for modulating the negative damping. It is shown in the paper that when the shaft is driving a extra load the shaft drawn power is more than the power responsible for destabilizing and it destabilizes the shaft causing shaft to whirl with very high amplitude.

A smart PID controller is designed using a Linear Quadratic regulator method. The input to the controller are the states which are measured from orthogonal vibrational velocity amplitude in a frame rotating with the shaft and the contrived negative internal damping is modulated with these signals. This way of controls lead to stable rotor even in presence of eccentricity and load. Further stabilization for vibration redemption is proposed for face abrasive tools which are often used for grinding and for finer surface preparation processes of flat surface of highly brittle material like inorganic glasses other such materials used in optoelectronic devices and preparation of semiconductor substrate or bases .The vibrations of surface abrasive tools often damage the material being processed. It is shown that active creation of negative damping in a rotating frame stabilizes the rotor a switching mechanism is further designed to prevent the reverse whirling of the rotor.

\section{REFERENCES}

[1] Meirovitch L. (1967). Analytical methods in vibration. N.Y Macmillan.

[2] Crandall SH, Karnopp DC, Kurtz DC. (1968). Dynamics of mechanical and electromechanical systems. McGraw Hill. https://doi.org/10.1007/978-3-642-20222-3_2

[3] Holmes R. (1972). Nonlinear performance of squeeze film bearings. Jr. of Mechanical Engineering Science 14(1): 74-77.

[4] Harnoy A. (1976). An analysis of stress relaxation in elastico-viscous hydrodynamic lubrication of sleeve bearings. ASLE Transactions 19: 301.

[5] Rosenberg RC, Karnopp D. (1983). Introduction to physical system dynamics. Mc. Graw Hill, New York.

[6] Mukherjee A. (1984). Effect of bi-phase lubricants on dynamics of rigid rotors. Transaction of ASME, Jr. of Lubrication $\quad$ Technology 105 : 2. https://doi.org/10.1115/1.3254539

[7] Baz A, Poh S. (1988). Performance of an active control system with Piezo electric actuators. Journal of sound and $\quad$ Vibration $326(2)$ : 324 https://doi.org/10.1016/0022-460x(88)90245-3

[8] Margolis DL, Karnopp DC, Rosenberg CR. (1990). System Dynamics a unified approach. John wiley and sons. https://doi.org/10.1109/TSMC.1976.4309434

[9] Halder B, Mukherjee A, Karmakar R. (1990). Theoretical and experimental studies on squeeze film stabilizer for flexible rotor-bearing systems using Newtonian and viscoelastic lubricants. Jr. of Vibration 
and Acoustics, Transaction of ASME 112(4): 473-482. https://doi.org/10.1115/1.2930131

[10] Burdess JS, Fawcett JN. (1992). Experimental evaluation of a piezoelectric actuator for the control of vibration in a cantilever beam. IMECHE. Part I: J. Sys. and control eng 206: 99-106. https://doi.org/10.1243/PIME_PROC_1992_206_315_0 2

[11] Preumont A, Dufour J, Malekian C. (1992). Active damping by a local force feed back with piezoactuatotrs. Journal of Guidence and Control 15(2): 390-395. https://doi.org/10.2514/3.20848

[12] Smith RD, Weldon WF. (1995). Nonlinear control of a rigid rotor magnetic bearing system modeling and simulation with full state feedback. IEEE transaction on Magnetics 31(2). https://doi.org/10.1109/20.364771

[13] Vaz JAC. (1995). Theoretical and experimental studies on the dynamics and control of intelligent beam structure with special reference to flexible manipulators $\mathrm{PhD}$ dissertation department of mechanical engineering. Indian Institute of Technology, 1995 Kheradpir, Pin 721 302, India.

[14] Bentley JP. (1995). Principle of measurement system. Third edition, Pearson.

[15] Murthy DVS. (2003). Transducer and Instrumentation. PHI, 2003

[16] Ballas RG. (2006). Piezoelectric Multilayer Beam Bending Actuators. Springer, ISBN 978-3-540-32641-

[17] Mukherjee A, Karmakar R, Samantaray AK. (2006). Bond graphs in modeling, simulation and fault identification. I. K. International Publishing House Pvt. Ltd. India, CRC Taylor and Francis, USA.

[18] Samantaray AK, Bhattacharyya R, Mukherjee A. (2006). An investigation in to the physics behind the stabilizing effect of two phase lubricants in journal bearings. Jr. of Vibration and Control 12(1): 425-442.
[19] Kirillov ON. (2007). Gyroscopic stabilization in the presence of non-conservative forces. Dokl. 76: 780-785. https://doi.org/10.1134/S1064562407050353

[20] Glebov VV, Sorokin VM, Puchkov VP, Ignat'ev DA. (2007). Eliminating vibrations in the face grinding of glass. Russian Engineering Research 27: 305-308. https://doi.org/10.3103/s1068798x07050164

[21] Bou-Rabee NM, Marsden JE, Romero LA. (2008). Dissipation-induced heteroclinic orbits in tippe tops. SIAM Review 50(2): 325-344. https://doi.org/10.2307/20454106

[22] Samantaray AK, Bhattacharyya R, Mukherjee A. (2008). On stability of Crandall gyro-pendulum. Physics Letters 372: $238-243$ https://doi.org/10.1016/j.physleta.2007.07.024

[23] Aurich A, Bierman D, Blum H, Brecher C, Carstensen C, Denkena B, Kloche F, Kroeger M, Steinmann P, Weiner K. (2009). Modelling and simulation of process machine interaction in grinding. Production Engineering Research and Development 3: 111-120. https://doi.org/10.1007/s11740-008-0137-x

[24] Simon A. (2009). Adaptive disturbance rejection and stabilization for rotor systems with internal damping. PhD. Dissertation Graduate Faculty of Auburn University Alabama US. https://doi.org/10.1115/DETC2009-86610

[25] Ortega AB, Carbajal FB, Navarro GS, Salazar MAO. (2018). Active vibration control of the rotor bearing system based on dynamic stiffness. Rev facts Aniv 2526.

[26] Active stabilization of rotors with circulating forces due to spinning dissipation. Journal of Vibration and Control 17: 1509-1524. (2010).

[27] A proposal for active control of face abrasive tool wandering. Journal of Vibration and Control 1077546311402708 (2011). 